\title{
Globe
}

Revue internationale d'études québécoises

\section{Michael Gauvreau et Ollivier Hubert [dir.] : The Churches and \\ Social Order in Nineteenth and Twentieth-Century Canada, Montréal, McGill-Queen's University Press, 2006}

\section{Dominique Marquis}

Volume 11, numéro 1, 2008

URI : https://id.erudit.org/iderudit/1000504ar

DOI : https://doi.org/10.7202/1000504ar

Aller au sommaire du numéro

Éditeur(s)

Globe, Revue internationale d'études québécoises

ISSN

1481-5869 (imprimé)

1923-8231 (numérique)

Découvrir la revue

Citer ce compte rendu

Marquis, D. (2008). Compte rendu de [Michael Gauvreau et Ollivier Hubert [dir.] : The Churches and Social Order in Nineteenth and Twentieth-Century Canada, Montréal, McGill-Queen's University Press, 2006]. Globe, 11(1), 289-292. https://doi.org/10.7202/1000504ar d'utilisation que vous pouvez consulter en ligne.

https://apropos.erudit.org/fr/usagers/politique-dutilisation/ 


\section{RECENSIONS}

Michel Lacroix [éd.]

\section{Michael Gauvreau et Ollivier Hubert [dir.] The Churches and Social Order in Nineteenth and Twentieth-Century Canada Montréal, McGill-Queen’s University Press, 2006.}

Lidée de publier ce recueil est née d'une volonté de renouveler les perspectives de recherche en histoire de l'Église canadienne. Parce qu'il leur apparaissait que cette dernière avait, durant les $\mathrm{XIX}^{e}$ et XXe siècles, multiplié les lieux d'exercice de son pouvoir, les directeurs du présent volume, Michael Gauvreau et Ollivier Hubert, ont invité plusieurs auteurs à réfléchir aux liens entre Église et contrôle social. Ils ont ainsi privilégié une approche plus sociale que théologique ou idéologique de l'histoire de l'Église et les résultats permettent de mieux cerner sa place au cœur des sociétés où elle agit. De plus, en présentant à la fois des études sur l'Église catholique et sur les Églises protestantes, les directeurs ont voulu replacer chaque démonstration dans une perspective "intra-chrétienne" plus riche que les approches dénominatives, géographiques ou nationales.

Gauvreau et Hubert présentent d'abord la problématique qui alimente tout le volume et ils appuient leur questionnement sur un bilan historiographique très détaillé, bilan qui pourrait aisément servir de modèle du genre. L'apport de la recherche au Canada anglais et au Canada français est souligné, mais le riche corpus européen, surtout britannique et français, est aussi mis à contribution afin de démontrer toutes les possibilités offertes par une nouvelle approche socioculturelle de l'histoire de l'Église.

Le premier chapitre lance la discussion autour d'un problème méthodologique: Christine Hudon et Ollivier Hubert soumettent les rapports annuels produits par des curés catholiques à un excellent exercice de 
critique des sources. Ils présentent les différentes utilisations possibles de ces documents qui, loin d'être parfaits, permettent néanmoins de "mesurer" le contrôle administratif exercé par les évêques sur leurs paroisses. Ils ne répondent peut-être pas directement au questionnement de départ, mais ils prouvent que les outils nécessaires à de telles analyses existent.

Par la suite, huit études démontrent toute la richesse d'un angle d'analyse qui ne présente pas l'Église comme un producteur exclusif de discours moral et idéologique. Si, de prime abord, un lien entre tous ces textes semble difficile à établir, il résulte toutefois de leur conjugaison que les Églises, quelles qu'elles soient, sont une composante essentielle du système de régulation sociale et qu'elles participent à l'intégration des individus au sein des communautés.

\section{Ainsi, Nancy Christie dans "Carnal Connection and Other} Misdemeanours", montre comment les cours religieuses locales établies par les communautés presbytériennes du Haut-Canada puis de l'Ontario sont utilisées comme agents de contrôle des comportements déviants. Elle souligne aussi qu'à la fin de la période étudiée, la "nationalisation" de l'Église presbytérienne et une certaine forme de privatisation des valeurs religieuses et morales diminuent considérablement l'influence de ces cours, établies principalement sur des valeurs communautaristes.

Hannah M. Lane aborde la question des différents modes de financement des Églises évangélistes du Nouveau-Brunswick et du Maine et souligne que ces multiples campagnes peuvent aisément devenir des facteurs d'exclusion des pauvres. En plus de faire la description minutieuse des modes de financement de ces Églises, son texte remet en question la qualité et la pertinence des sources, qui doivent être utilisées avec précaution parce qu'elles ne reflètent pas toujours l'exacte situation économique d'une communauté.

La question de l'autorité cléricale et de sa contestation est abordée par Kenneth L. Draper dans un texte où il évoque trois histoires exemplaires "qui finissent mal". Le récit de trois pasteurs qui voient leur autorité contestée par leurs paroissiens devient l'occasion de montrer que ces relations parfois tendues ne sont pas le signe d'une diminution de la ferveur religieuse, mais plutôt l'indice d'une volonté de rehausser la qualité de l'encadrement religieux afin de mieux distiller l'esprit chrétien au sein de toute la communauté.

L'éducation et la charité sont aussi des lieux où l'Église catholique a exercé un contrôle important au Québec. Ces dimensions sont étudiées par Brigitte Caulier, Jean-Marie Fecteau et Éric Vaillancourt dans deux textes qui 
constituent d'excellents exemples de la portée de l'engagement de l'Église québécoise comme institution sociale. Brigitte Caulier propose une analyse du contenu des programmes scolaires catholiques et protestants, du début du $\mathrm{XX}^{\mathrm{e}}$ siècle aux années 1960 , où se dessinent deux visions du monde distinctes. Son étude démontre avec force que les programmes protestants sont beaucoup mieux adaptés aux réalités changeantes de la société québécoise et que leur contenu est axé sur la formation citoyenne et une plus grande tolérance, alors que des programmes catholiques se dégage une volonté très nette d'appliquer les principes catholiques à toutes les facettes de l'éducation. Fecteau et Vaillancourt, quant à eux, examinent l'implantation de la Société Saint-Vincent de Paul à Montréal au milieu du XIX siècle et la manière dont cette association charitable a réussi à accueillir les laïques au sein des réseaux paroissiaux et à leur donner un véritable rôle d'intermédiaires entre les familles et les institutions cléricales.

L'intégration des ouvriers et des nouveaux arrivants fait l'objet de deux analyses. Dans "Factories and Foreigners 1890-1930" Michael Gauvreau souligne que la dichotomie entre classes moyennes et ouvrières au sein de l'Église n'est plus de mise : les nouvelles études démontrent clairement que les classes ouvrières n'ont pas été soumises aux classes moyennes, mais qu'elles ont développé leur propre culture religieuse, qui répond à des besoins précis. Dans le même ordre d'idées, Roberto Perin brosse un portrait révélateur des moyens pris par les différentes Église torontoises pour faciliter l'intégration des immigrants entre 1947 et 1965 , moyens qui dépassent la pratique religieuse pour investir massivement le champ du social.

Finalement, Gilles Routhier analyse la préparation, la réception et le suivi du Concile Vatican II par les évêques canadiens. Il met en évidence le caractère distinct du Québec à cet égard en montrant que dans leurs entreprises de renouvellement de la pastorale et des institutions ecclésiales, les évêques ont été influencés autant par Vatican II que par le mouvement sociopolitique plus vaste créé par la Révolution tranquille. Cet article correspond peut-être moins à la problématique générale du livre, mais il demeure très intéressant de constater que l'Église catholique n'est pas homogène et que les solutions à différents problèmes sont toujours élaborées par ses représentants régionaux en tenant compte des conditions particulières à chaque société. Cela montre comment l'Église catholique cherche à prendre place dans le milieu social, culturel, politique, voire économique, dans lequel elle agit. 
Il n'est jamais aisé de diriger un ouvrage collectif, mais Michael Gauvreau et Ollivier Hubert ont su regrouper des articles qui, tout en abordant plusieurs horizons, mettent en lumière les différents modes d'insertion des Eglises, catholiques et protestantes, dans un vaste système de régulation sociale. Certains textes ont, à mon avis, mieux répondu aux questionnements suggérés en introduction, mais tous, sans exception, contribuent à ce renouvellement des perspectives tant souhaité par les directeurs de l'ouvrage. Ni les catholiques ni les protestants ne pourront déplorer leur faible représentation dans cet ouvrage. Toutefois, je regrette pour ma part qu'une réelle approche comparative n'y ait pas été réalisée; exception faite de l'étude de Brigitte Caulier, les auteurs laissent au lecteur le soin d'établir ses propres critères de comparaison. Le caractère "intrachrétien " n'est donc déterminé ici que par la présence, dans le même ouvrage, d'études sur des sujets catholiques ou protestants, mais rarement catholiques et protestants.

Dominique Marquis

Université du Québec à Montréal

\section{Serge Gagnon}

Quand le Québec manquait de prêtres.

La charge pastorale au Bas-Canada

Québec, Presses de l'Université Laval, 2006.

Ce livre de Serge Gagnon met à l'avant-scène les curés et vicaires, des années 1790 à 1830 . À travers ses six chapitres, l'auteur s'attarde aux composantes essentielles de leur tâche - le catéchisme, la prédication et la confession -, qui sont au cœur de la stratégie d'évangélisation.

À quoi servirait, se demande-t-il, la profusion d'eau bénite et autres activités rituelles si ces déploiements gestuels se déroulaient devant des assemblées ignorantes de ce qu'il faut croire et faire pour éviter l'enfer, aller au paradis ou séjourner au purgatoire avant d'atteindre le lieu de l'éternel bonheur (p. 4-5)?

Comme l'indique son titre, l'ouvrage cherche tout particulièrement à illustrer les effets de la pénurie de prêtres qui sévit à l'époque. Les nombreux exemples et les longues citations qui appuient la démonstration visent à mettre en relief la complexité du travail pastoral, de la gestion épiscopale et, surtout, à restituer l'humanité des acteurs sociaux en faisant 